\title{
Predação de ovos de Corythomantis greeningi Boulenger, 1896 (Anura, Hylidae) por Solenopsis invicta Buren, 1972 (Formicidae: Myrmicinae)
}

\author{
Geane Limeira da Silva ${ }^{1}$ \\ Ednilza Maranhão dos Santos ${ }^{1 *}$ \\ Juliana Pessoa Gomes ${ }^{2}$ \\ ${ }^{1}$ Universidade Federal Rural de Pernambuco, Unidade Acadêmica de Serra Talhada \\ Fazenda Saco, s/n., CEP 59000-000, Serra Talhada-PE, Brasil \\ ${ }^{2}$ Universidade Federal de Pernambuco, Centro de Ciências Biológicas \\ Departamento de Zoologia, Recife-PE, Brasil \\ *Autor para correspondência \\ ednilzamaranhao@yahoo.com.br
}

Submetido em 16/03/2010

Aceito para publicação em 02/08/2010

\section{Resumo}

O presente trabalho vem documentar através de um evento ocasional a predação de ovos da rã Corythomantis greeningi por formigas da espécie Solenopsis invicta em borda de córrego temporária em 29 de março de 2009, na localidade Olho d’Água, município de Santa Cruz da Baixa Verde, Pernambuco.

Unitermos: formiga, Nordeste, ovos de anura, predação

\section{Abstract}

Predation of eggs of Corythomantis greeningi Boulenger, 1896 (Anura, Hylidae) by Solenopsis invicta Buren (Formicidae: Myrmidinae). This work is documented through an occasional event of predation of eggs of the frog Corythomantis greeningi by ants of the species Solenopsis invicta at the edge of a temporary brook on 29 March 2009, in the town of Olho d'Água, municipality of Santa Cruz da Baixa Verde, Pernambuco.

Key words: ant, eggs of anuran, Northeast, predation

A predação de anuros por invertebrados vem sendo documentada por vários autores, que destacam como predadores principais desse grupo, aranhas e insetos (Villa et al., 1982; Duellman e Trueb, 1994; Downiet et al., 1995; Bernarde et al., 1999; Pombal, 2007; Santos, 2009). De uma maneira geral, registros de predação de anuros por invertebrados são mais comuns entre a fase adulta e a larval, do que na fase de embrião (Downiet et al., 1995; Santos, 2009). Informações sobre predação de ovos de anuros por formigas ainda são incipientes (Sazima, 1972; Prado et al., 2005; Lingnau e DiBernardo, 2006).

Corythomantis greeningi é um anfíbio anuro, pertencente à família Hylidae, que apresenta distribuição restrita às regiões xéricas e sub-úmidas do Brasil, 
amplamente distribuída no Bioma Caatinga (Rodrigues, 2003). Possui cabeça bem ossificada, em formato de capacete ou escudo que tem a função de tampa em fendas rochosas, servindo de proteção contra predadores; ocupam ambientes como bromeliáceas, ocos de arvores e frestas de rochas (Jared et al., 1999; Navas et al., 2002). Esta espécie tem sua reprodução bem marcada na época chuvosa, ocupando ambientes aquáticos temporários, lóticos e rasos. Machos dessa espécie utilizam como sítio de canto frestas de rochas para atrair fêmeas e seus girinos podem ser observados em pequenas poças ao longo do leito de corpos d'água temporários (Juncá et al., 2008).

Solenopsis invicta é um himenóptero da família Formicidae, conhecida como formiga lava-pé, é uma espécie nativa da América do Sul que ocorre como invasora nas Américas Central e do Norte, Ásia e Oceania (Holway et al., 2002; Zhang et al., 2007). Essa formiga é conhecida pela sua agressividade e ferroada dolorosa podendo provocar processos alérgicos (Fowler et al., 1990). Solenopsis invicta está entre os predadores mais ativos e abundantes, causando prejuízo em muitas culturas agrícolas (Banks et al., 1990; Williams et al., 2001; Jetter et al., 2002). Por ser uma voraz predadora, esta espécie é candidata ao uso como agente de controle biológico (Eubanks, 2001). A formiga lava-pé também é apontada por vários autores como provável responsável pelo decréscimo da diversidade de artrópodes nativos em diversos ecossistemas invadidos, também causando danos a outros organismos (Wojcik et al., 2001; Jetter et al., 2002; Morrison, 2002; Parris et al., 2002). Segundo Fowler et al. (1991), o padrão alimentar de formigas é constituído basicamente por proteínas, carboidratos e lipídeos, sendo que as proteínas seriam adquiridas por meio da predação de outros insetos e demais invertebrados. Solenopsis invicta foi observada alimentando-se de invertebrados (Eubanks et al., 2002) e vertebrados, como ovos de serpentes, lagartos e ovos e filhotes recém-nascidos de aves (Teixeira et al., 1991; Conners, 1998; Chalcraft e Andrews, 1999). Registros de predação de ovos de anuros por formigas desse gênero foram citados para Physalaemus cuvieri (Sazima, 1972) e $P$. aff. gracilis (Lingnau e Di-Bernardo, 2006).

Em 29 de março de 2009, às 9h, na localidade Sítio Olho d'Água $\left(07^{\circ} 85^{\prime} 8,90^{\prime}\right.$ 'S/038¹7’7,09”W), município de Santa Cruz da Baixa Verde, Pernambuco, foram registradas formigas forrageando, transportando e alimentando-se de ovos de $C$. greening $i$ na borda de um córrego (Figura 1). Esse córrego possui cerca de $2 \mathrm{~m}$ de largura e cerca de $2 \mathrm{~km}$ de comprimento dentro da localidade. A desova possuía mais de 700 ovos e estava na borda do corpo d'água, sobre lajedo. $\mathrm{O}$ evento foi documentado através de câmera fotográfica e as imagens estão depositadas na coleção Herpetológica da Universidade Federal Rural de Pernambuco (UFRPE), Unidade Acadêmica de Serra Talhada. Exemplares da formiga $(n=4)$ foram coletados com pinça, colocadas em um recipiente de vidro com álcool a $70 \%$ e enviados ao laboratório de Taxonomia e Ecologia de Insetos da Universidade Federal de Pernambuco (UFPE) para identificação. Os exemplares foram tombados na Coleção Entomológica da UFPE (números dos tombamentos: 1004HF, 1005HF, 1006HF e 1007HF). O presente trabalho faz parte de uma pesquisa mais ampla sobre a anurofauna local. O anuro e a desova foram identificados com base na literatura específica (Jared et al., 1999; Navas et al., 2002; Juncá et al., 2008) e ajuda de especialistas.

O risco de predação parece ser maior na água (Pombal, 2007; Santos, 2009) do que no ambiente terrestre, como já relatado por Lingnau e Di-Bernardo (2006). Esses autores registraram formigas, do gênero Solenopsis, predando ovos de anuros que apresentavam desova em ninho de espuma, sugerindo que esse tipo de ninho não é completamente eficiente contra alguns predadores; todavia, destacaram que as formigas se alimentaram dos ovos mais superficiais do ninho. No presente relato foi possível observar formigas sobre a desova, transportando e se alimentando dos ovos localizados na camada mais externa da desova. Estes ovos estavam fora d'água, o que possivelmente facilitou o ataque das formigas, entretanto para avaliar melhor o custo desse evento seria importante um estudo mais direcionado. Esse é o primeiro registro de predação de ovos de anuros por S. invicta na região Nordeste, embora já tenha sido registrada a predação de anuros pelo gênero Solenopsis em outras regiões do Brasil (Sazima, 1972; Lingnau e Di-Bernardo, 2006), ainda não se sabe o real impacto desse grupo como potencial predador de anuros. 


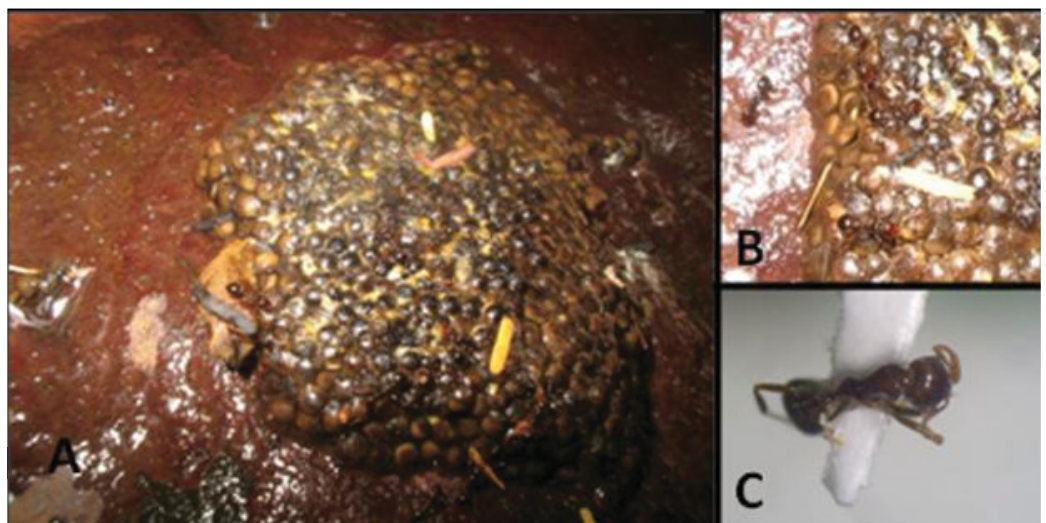

FIGURA 1: Imagens da predação da desova de Corythomantis greeningi por Solenopsis invicta em 29/ III/2009 na localidade Olho d'Água, Santa Cruz da Baixa Verde, Pernambuco. A - Imagem geral da desova; B - Imagem ampliada de algumas formigas sobre a desova; C - Espécime de S. invicta coletado.

\section{Agradecimentos}

À equipe de herpetologia da Unidade Acadêmica de Serra Talhada pela ajuda durante as atividades de campo. Ao proprietário Sr. Antônio Timóteo pela autorização de coleta de dados na sua localidade, ao Programa de Iniciação Científica (Pibic) pela bolsa concedida ao primeiro autor e ao IBAMA pela licença n. 11218-1.

\section{Referências}

Banks, W. A.; Adams, C. T.; Lofgren, C. S.; Wojcik, D. P. 1990. Imported fire ant infestation of soybean fields in the southern United States. Florida Entomologist, 73 (3): 503-504.

Bernarde, P. S.; Souza, M. B. de; Kokubum, M. N. de C. 1999. Predation on Hyla minuta Peters, 1872 (Anura, Hylidae) by Ancylometes spp. (Araneae, Pisauridae). Biociências, 7 (1): 199203.

Chalcraft, D. R.; Andrews, R. M. 1999. Predation on lizard eggs by ants: species interactions in a variable physical environment. Oecologia, 119: 285-292.

Conners, J. S. 1998. Opheodrys aestivus (rough green snake). Egg predation. Herpetological Review, 29: 243.

Downiet, J. R.; Disney, R. H. L.; Collins, L.; Hancock, E. G. 1995. A new species of Megaselia (Dipera, Phoridae) whose larvae prey upon the eggs of Leptodactylus fuscus (Anura, Leptodactylidae). Jounal of Natural History, 29: 993-1003.

Duellman, W. E.; Trueb, L. 1994. Biology of amphibians. McGraw-Hill Book Company, New Cork, USA, 670pp.

Eubanks, M. D. 2001. Estimates of the direct and indirect effects of red imported fire ants on biological control in field crops. Biological Control, 21: 35-43.

Eubanks, M. D.; Blackwell, S. A.; Parrish, C. J.; Delamar, Z. D.; Hull-Sanders, H. 2002. Intraguild predation of beneficial arthropods by red imported fire ants in cotton. Environmental Entomology, 31 (6): 1168-1174.

Fowler, H. G.; Bernardi, S. V. E.; Delabie, J. H. C.; Forti, L. C.; Silva, V. P. 1990. Major ant problems of South America. In: Vandermeer, R. K.; Jaffé, K. \& Cedeño, A. (Eds). Applied Myrmecology: a world perspective. Westview Press, Califórnia, USA, p.3-14.

Fowler, H. G.; Forti, L. C.; Brandão, C. R. F.; Delabie, J. H. C.; Vasconcelos, H. L. 1991. Ecologia nutricional de formigas. In: Panizzi, A. R. \& Parra, J. R. P. (Eds). Ecologia nutricional de insetos e suas implicações no manejo de pragas. Manole, São Paulo, Brasil, p.131-223.

Holway, D. A.; Lach, L.; Suarez, A. V.; Tsutsui, N. D.; Case, T. J. 2002. The causes and consequences of ant invasions. Annual Review of Ecology and Systematics, 33: 181-233.

Jared, C.; Antoniazzi, M. M.; Katchburian, E.; Toledo, R. C.; Freymuller, E. 1999. Some aspects of the natural history of the casque-headed tree tree-frog Corythomantis greeningi Boulenger (Hylidae). Annales Des Siences Naturelles Biologie Animale et Zoologie, 1999 (3): 105-115.

Jetter, K. M.; Hamilton, J.; Klotz, J. H. 2002. Red imported fire ants threaten agriculture wildlife and homes. California Agriculture, 56 (1): 26-34.

Juncá, F. A.; Carneiro, M. C. L.; Rodrigues, N. N. 2008. Is a dwarf population of Corythomantis greeningi Boulenger, 1896 (Anura, Hylidae) a new species? Zootaxa, 1686: 48-56.

Lingnau, R.; Di-Bernardo, M. 2006. Predation on foam nests of two Leptodactylid frogs by Solenopsis sp. (Hymenoptera, Formicidae) and Liophis miliaris (Serpentes, Colubridae). Biociências, 14 (2): 223-224.

Navas, C. A.; Jared C.; Antoniazzi, M. M. 2002. Water economy in the casque-headed tree-frog Corythomantis greeningi (Hylidae): role of behaviour, skin, and skull skin co-ossification. Journal of Zoology, 257: 525-532.

Morrison, L. W. 2002. Long-term impacts of an arthropodcommunity invasion by the imported fire ant, Solenopsis invicta. Ecology, 83 (8): 2337-2345. 
Parris, L. B.; Lamont, M. M.; Carthy, R. R. 2002. Increased incidence of red imported fire ant (Hymenoptera: Formicidae) presence in loggerhead sea turtle (Testudines: Cheloniidae) nests and observations of hatchling mortality. Florida Entomologist, 85 (3): 514-517.

Prado, C. P. A.; Toledo, L. F.; Zina, J.; Haddad, C. F. B. 2005.Trophic eggs in the foam nests of Leptodactylus labyrinthicus (Anura, Leptodactylidae): an experimental approach. Herpetological Journal, 15: 279-284.

Pombal-Jr, J. P. 2007. Notas sobre predação em uma taxocenose de anfíbios anuros no sudeste do Brasil. Revista Brasileira de Zoologia, 24 (3): 841-843.

Rodrigues, M. T. 2003. Herpetofauna da Caatinga. In: Tabarelli, M. \& Silva, J. M. C. (Eds). Biodiversidade, ecologia e conservação da Caatinga. Universidade Federal de Pernambuco, Recife, Brasil, p.181-236.

Santos, E. M. 2009. Notas sobre predação de anuros em uma poça temporária no nordeste do Brasil. Boletim do Museu de Biologia Mello Leitão (N. Sér.), 25: 77-82.
Sazima, I. 1972. Predadores do anuro Physalaemus curievi na Cidade Universitária "Armando de Salles Oliveira" São Paulo. Ciência e Cultura, 24 (6): 1972.

Teixeira, D. M.; Luigi, G.; Schloemp, I. 1991. Aves brasileiras como presas de atrópodes. Ararajuba, 2: 69-74.

Villa, J.; Mcdiarmid, R. W.; Gallardo, J. M. 1982. Arthropod predators of Leptodactylid forg foam nests. Brenesia, 19 (20): $577-$ 589.

Williams, D. F.; Collins, H. L.; Oi, D. H. 2001. The red imported fire ant (Hymenoptera: Formicidae) an historical perspective of treatment programs and the development of chemical baits for control. American Entomologist, 47 (3): 146-159.

Wojcik, D. P.; Allen, C. R.; Brenner, R. J.; Forys, E. A.; Jouvenaz, D. P.; Lutz, R. S. 2001. Red imported fire ants: impact on biodiversity. American Entomologist, 47 (1): 6-23.

Zhang, R.; Li, Y.; Liu, N.; Porter, S. D. 2007. An overview of the red imported fire ant (Hymenoptera: Formicidae) in mainland China. Florida Entomologist, 90: 723-731. 